\author{
Anna DUTKA-MAŃKOWSKA \\ Uniwersytet Warszawski \\ a.mankowska@uw.edu.pl
}

\title{
QUELQUES CONTRAINTES DANS LA TRADUCTION DU DISCOURS DIRECT DU FRANÇAIS EN POLONAIS : LE CADRAGE ÉNONCIATIF ET LE CONNECTEUR MAIS AU DÉBUT D'UNE RÉPLIQUE
}

\section{INTRODUCTION}

Nous voudrions présenter quelques cas de contraintes qui s'observent dans la traduction du discours direct $d u$ français vers le polonais. Nous envisageons les séquences dialogales dans le discours littéraire, et en particulier le verbe neutre dire, dans le discours citant et le connecteur mais, au début d'une réplique.

Nous admettons que des "paramètres culturels qui se reflètent dans l'organisation collective du discours » (Guillemin-Flescher 2003 : 9 sv) permettent une analyse des textes parallèles et comparables. Certains phénomènes (p. ex. l'explicitation) reconnus comme propres au processus de traduction, relèvent également des contraintes propres à la culture d'accueil (Becher 2010 : 14), y compris des stratégies dans la construction $\mathrm{du}$ discours et des tendances stylistiques. D'autre part, «la représentation ordinaire» (Chevalier, Delport 1995 : 9) que le traducteur se fait du monde représenté dans le texte qu'il traduit, basée sur des phénomènes cognitifs, est responsable des ressemblances entre les traductions d'un texte vers diverses langues.

Dans une perspective contrastive anglais-français, L. Gournay (2013) a montré en français une relation très étroite entre l'incise et le discours cité, et elle a plaidé pour une séparation nette en français entre les paroles du personnage et le récit. Nous montrerons que le choix des verbes de dire dans l'incise dans la traduction en polonais s'appuie sur une 
interprétation du sens dans le discours cité, laquelle favorise une plus grande homogénéité. Le passage vers le polonais, contrairement au texte de départ, témoigne des liens entre les paroles des personnages et le récit.

En ce qui concerne des répliques introduites par mais, la reconstruction en polonais se fait avec des moyens linguistiques très diversifiés, souvent sans un équivalent de ce marqueur. Des expressions situées dans l'énoncé concourent à modifier le sens en présentant une opposition renforcée, et même un affrontement, ou, au contraire, une opposition faible.

Dans deux premières parties nous admettons l'approche linguistique et dans la partie finale nous présenterons les résultats compte tenu de la créativité du traducteur.

Les textes traduits ont été publiés dans la deuxième moitié du $X X^{\mathrm{e}} \mathrm{s}$. et au début du XXI ${ }^{\mathrm{e}} \mathrm{s}$. Nous considérons qu'ils sont soumis aux mêmes contraintes, même si les textes de départ, retenus pour les phénomènes étudiés, datent aussi du XIX ${ }^{\mathrm{e}} \mathrm{s}$.

\section{LE CADRAGE ÉNONCIATIF - LE DISCOURS CITANT DANS LA TRADUCTION}

\subsection{LE CHOIX DU VERBE DE DIRE DANS LES INCISES}

Nous envisageons les cas où le verbe dire n'est pas traduit par son équivalent lexical - un verbe de base mówić/powiedzieć/rzec.

Les traits sémantiques des verbes introducteurs peuvent être décrits dans ce cas comme qualifiant l'acte de parole, et cette qualification peut être ajoutée au prédicat, p. ex. un adverbe de manière, ou bien intégrée au prédicat (Hanote 2004 : 541). Les verbes introducteurs constituent « un commentaire (modal) émanant de l'énonciateur-origine» (Poncharal 2003 : 86), un commentaire qui porte sur la prise de parole et qui est pris en charge par l'énonciateur rapporteur. Le contenu du discours cité est relié à la situation d'énonciation rapportée ou au récit où elle figure. Ainsi le DCnt ${ }^{1}$ oriente-t-il la lecture du DCité. $^{2}$

1 Abréviations : DCnt - discours citant, DCité - discours cité, TD - texte de départ, TA - texte d'arrivée.

2 A. Rabatel (2014) postule de distinguer le sujet modal qui est une instance de validation et l'instance de la prise en charge ; ces instances divergent dans le discours. Nous ne suivrons pas cette proposition. 
La position finale ou imbriquée du DCnt est qualifiée par Danon-Boileau (1982 : 64) comme descriptive : l'énoncé citant ne fait que qualifier le DCité et il n'est pas asserté (il dépend du DCité). Postposé, le verbe dire ne sert plus à construire une occurrence de parole, il identifie seulement la source énonciative rapportée. Par contre, les verbes sémantiquement riches contribuent au sens du DCité et ils explicitent l'attitude du locuteur cité.

Nous avons constaté (voir aussi Dutka-Mańkowska 2010) qu'en TA dominent les verbes qui qualifient les relations entre les énonciateurs et l'évaluation de l'énonciateur citant de l'échange en cours, p. ex. odeprzeć, odpowiedzieć, odrzec (répondre, répliquer), spytać (interroger), zwrócić się, zagadnać (s'adresser), odezwać się (prendre la parole), et de la manière de dire (verbes suprasegmentaux), p. ex. krzyczeć (crier), jęknać (gémir), mruknać (grommeler), pisnać (piailler, crisser), zawołać (appeler, hurler), wypalić (dire brusquement).

Les verbes d'action sont peu nombreux (ils décrivent les gestes, l'expression du visage qui accompagnent les mots) : skrzywić się (faire une grimace), wzdrygną́ się (sursauter).

Certains auteurs soulignent l'impact pragmatique des verbes sémantiquement plus riches dans le TA. P. ex. les verbes directifs, servent à ménager la force illocutoire des actes de langage. J. Bourne (2002) se réfère aux règles de politesse dans les deux langues, ou plutôt à l'idée que le traducteur se fait des attentes du lecteur. Il explique la variété des verbes de dire par la volonté du traducteur de s'exprimer d'une manière précise, variée, mais aussi d'aboutir à un effet pragmatique : il s'agit d'interpréter l'interaction. Mais dans le dialogue romanesque, c'est la dynamique narrative qui compte et non pas le principe social de politesse (Durrer 1994 : 109), donc il faut être attentif à la relation entre les paroles des personnages et le récit (point 1.2 ci-dessous). L'incise non seulement explicite le sens (reprise, réplique, réponse, question), mais aussi la manière de parler (répétition, gémissement, cri).

On a une mise en scène des propos rapportés. Il faut souligner que l'interprétation et la reconstruction dans le TA repose sur la manière dont le traducteur conceptualise le lecteur virtuel ou bien des principes esthétiques de l'écrivain. Ainsi la première traduction de Madame Bovary en polonais donne beaucoup de verbes de base (mówić/powiedzieć/rzec), alors que la seconde possède beaucoup de verbes sémantiquement riches. La mise en scène du discours dans le deuxième cas est bien plus variée, 
mais elle est contraire au projet esthétique de Flaubert d'un narrateur invisible (Dutka-Mańkowska 2012).

Comme J.-Cl. Chevalier et M.-F. Delport (1995 : 47-48), nous admettons que les modifications dans la traduction du discours direct (DCité et DCnt) peuvent être envisagées comme explicitation (si le traducteur «met au jour des informations contenues dans la situation qu'évoque la phrase à traduire») ou bien comme amplification (lorsqu'il «ajoute, il développe, au gré de ce que son imagination lui présente»33).

Il est à noter que l'explicitation peut se faire au prix d'une redondance, qui montre d'autant mieux le lien entre le DCité et le DCnt :

A propos, je voulais te demander - la voix d'Olga se teinta d'une nonchalance appuyée. Cette infusion que je t'ai conseillée, ça a de l'affet sur tes insomnies? (Crime 118-119)

$\left(1^{\prime}\right)^{4} \quad$ A przy okazji chciałam cię spytać, czy dobrze sypiasz po ziółkach, które ci poleciłam - spytała Olga od niechcenia. (Zbrodnia 104) 'demanda Olga avec nonchalance'

Dans (1'), le verbe spytać 'demander' est repris dans DCnt.

Le verbe dans l'incise relève souvent d'une explicitation. Le traducteur mentionne le fait énonciatif en polonais. C'est une tendance forte, comme dans ( $\left.2^{\prime}\right)$, avec des expressions qui dans le TD donnent des circonstances de l'acte d'énonciation :

(2) Le docteur répondit d'une voix distraite : «Je suis bien content, mon petit...» et soudain : «Comment l'as-tu connue ?» (Désert 857)

(2') Doktor odpowiedział roztargnionym głosem: - To mnie bardzo cieszy, mój mały... - i nagle dodał: - Jak zawałeś tę znajomość? (Pustynia 151)

Dans (3'), le verbe rozprawiać 'délibérer' précise la manière de parler. Dans le DCité, l'accent d'insistance frappe le début de l'énoncé (les environs proches) et marque l'opposition par un mot vieilli, wszelako 'pourtant' :

3 D'autres auteurs donnent d'autres définitions de ces phénomènes ; les deux phénomènes consistent à rendre le texte plus long.

4 (1') se lit: la traduction de l'exemple (1). 
(3) - Du reste, disait l'apothicaire, l'exercice de la médecine n'est pas fort pénible dans nos contrées ; (Madame 87)

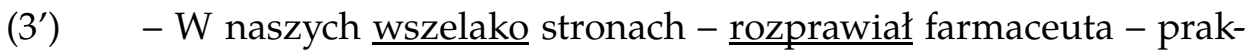
tyka lekarska nie jest zajęciem zbyt uciążliwym; (BovE 81) 'dans nos contrées pourtant - délibérait l'apothicaire - l’exercice de la médecine n'est pas fort pénible'

Le TD n'a pas d'expression vieillie ni de verbe du type délibérer et l'ordre des mots est canonique.

\subsection{LA RELATION ENTRE LES PAROLES DES PERSONNAGES ET LE RÉCIT}

Est-ce que la cohérence entre le DCité et le DCnt, que nous venons de présenter, influe sur la perméabilité entre le récit et l'incise ? Selon Gournay (2013), il y a de ce point de vue une différence fondamentale entre le français, où l'incise est en rupture par rapport au récit, et l'anglais, ou la prise de parole peut être coordonnée à une action au niveau du récit. Examinons deux cas en polonais :

\subsubsection{LA COORDINATION D'UN VERBE DE DIRE AVEC UN VERBE D'ACTION}

Une telle coordination est possible, ce qui n'est pas le cas en français (Dutka-Mańkowska 2010). Il en résulte que deux schémas de traduction d'une incise sont attestés, lorsqu'en TD une qualification du dire est ajoutée au prédicat : a) sans coordination et b) avec coordination, p. ex. :

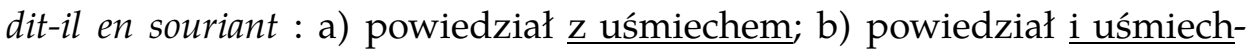
nał się.

La traduction sans coordination du verbe de dire est moins fréquente :

(4) - Il faut que je file au tribunal - dit-il en se levant. [...] (Le secret 332)

(4') - Muszę już pędzić do sądu - powiedział Vincent, podrywajac się. [...] ( Sekret 234)

La traduction avec le verbe de dire coordonné à celui d'action, fréquente, prouve que le polonais permet de ne pas séparer les paroles du personnage du récit :

(5) - Bonsoir, bébé, dit Fauchery, qui, familièrement, la baisa. (Nana fr 123) 
(5') - Dobry wieczór, bobo - powiedział Fauchery i pocałował ja poufale. (Nana pl 98) 'dit Fauchery et la baisa familièrement'

(6) - Excusez-moi, les garçons - dit-il en se levant. (Le secret 49)

(6') - Przepraszam was, chłopcy - powiedział i wstał. (Sekret 36) 'dit-il et il se leva'

Il peut arriver que la traduction sépare le verbe de dire et une action qui relève du paraverbal déplacée dans le récit est coordonnée avec une autre action :

(7) - Attends, mon chéri, dit-elle en le rejoignant.

Elle se planta juste devant lui [...] (Le secret 484)

$\left(7^{\prime}\right) \quad$ - Poczekaj, kochanie - powiedziała.

Podeszła i stanęła tuż przed nim ze smutnym uśmiechem. (Sekret 340)

1.2.2. UNE QUALIFICATION DU DIRE ENRICHIE PAR DES ÉLÉMENTS PUISÉS DANS LE RÉCIT

(8) [Il faut s'arrêter là où nous sommes arrivés...]

Elle ramena sa veste sur elle-même, la ferma, l'étriqua sur elle, fut reprise du même gémissement sauvage.

- C'est impossible, dit-elle. (Moderato fr 83)

(8') Włożyła żakiet, zapięła go, obciągnęła na sobie.

- To niemożliwe - powiedziała z przejmujacym zwierzęcym jękiem. (Moderato pl 117)

(9) Hector écoutait religieusement. Il posa une question.

- Et Nana, l'étoile nouvelle, qui doit jouer Vénus, est-ce que tu la connais ? (Nana fr 6)

$\left(9^{\prime}\right) \quad$ Hektor słuchał uważnie.

- A znasz Nanę, nową gwiazdę, która ma grać Wenus? - zapytał. (Nana pl 6)

\subsubsection{LA RÉDUCTION OU L'OMISSION DE L'INCISE}

Le cadrage énonciatif introduit par le DCnt se trouve souvent réduit dans le passage vers le polonais, en particulier le pronom qui 
réfère au co-énonciateur est effacé, ce qui montre que c'est la situation d'énonciation qui prime la construction de la référence.

(10) - Tu sais, lui dit-elle lentement, c'est une vraie malédiction... (Le secret 16)

$\left(10^{\prime}\right)$ - To chyba jakaś klątwa - powiedziała Ø Klara, cedząc słowa. (Sekret 12)

En français, l'expression qui ouvre la réplique donne le pronom de la deuxième personne, repris par le pronom de la troisième personne dans l'incise. En polonais, la particule de l'oral tu sais (Kerbrat-Orecchioni 2005 : 49-50), et plus précisément un marqueur d'appel à l'écoute (Dostie 2004 : 47), est effacée et dans l'incise il n'y a pas de pronom qui réfère au co-énonciateur.

L'incise peut être jugée superflue par le traducteur et effacée dans la traduction :

- Tiens - $\underline{\text { dit-elle }}$ - vous êtes rentré ? (Le désert 126)

- To ty wróciłeś? Ø (Pustynia 99)

Il faut souligner que l'usage de ne pas répéter les incises résulte des normes collectives. Comme l'ont montré Mossberg et Jansson Ghadiri (2010), en suédois la répétition des incises du type dit-il est de règle. Par contre, en polonais l'usage est différent.

La deuxième partie de notre article concerne un problème ponctuel dans le DCité : la traduction d'un 'mot du discours' au début d'une réplique.

\section{LE MARQUEUR ${ }^{5}$ MAIS AU DÉBUT D’UNE RÉPLIQUE}

Selon O. Ducrot (1980 : 92), mais introduit une opposition « aussi bien personnelle qu'intellectuelle». Dans le premier cas qui nous intéresse, on observe deux tendances dans sa traduction : celle à supprimer le marqueur et celle à intensifier ou à réduire l'opposition exprimée dans la réplique. Pour expliquer ces phénomènes nous avons approfondi l'examen des équivalents de mais dans le discours en position initiale

5 Nous adoptons la terminologie de Dostie (2004 : $40 \mathrm{sv})$. 
(Dutka 1992, Dutka 1993) : ale est un marqueur aussi polyvalent que mais, ależ peut se trouver uniquement au début d'une réplique, il exprime une opposition vive ou bien il intensifie le jugement à propos de l'argument introduit, lecz marque une opposition intellectuelle et il n'est pas approprié aux contextes où les interlocuteurs s'affrontent.

Nous avons emprunté à J.-Cl. Anscombre (2009) la notion d'attitude énonciative et nous avons considéré mais comme un marqueur mais de l'attitude énonciative d'opposition. Nous avons admis, à l'instar de l'auteur sus-mentionné, que les éléments initiaux comme mais ouvrent des espaces discursifs qui signalent que l'énoncé est censé se dérouler dans le cadre d'une opposition.

Cette optique nous a permis de montrer, dans une étude contrastive (Dutka-Mańkowska sous presse), que les phénomènes constatés s'expliquent par des contraintes de discours. En ce qui concerne la traduction, ces contraintes influent sur les choix des traducteurs, mais ceux-ci proposent aussi des solutions créatives ou opèrent certains choix. ${ }^{6}$

\subsection{LA TENDANCE À PLACER LE MARQUEUR MAIS AU DÉBUT DE LA RÉPLIQUE EN FRANÇAIS}

Souvent dans la traduction vers le polonais mais n'est pas traduit et on peut parler d"'effacement' (une opération de traduction qui vise à alléger le texte, Ballard 2003 : 49). Nous avons vérifié si, dans la traduction en sens inverse, vers le français, mais est un équivalent d'une expression polonaise dans le texte de départ. Il s'avère que mais est le plus souvent ajouté :

- Ø Co pan tu robi?! - w głosie kobiety drżała tajona furia. (co 'que' porte l'accent d'insistance) (Złoty 221)

Mais que faites-vous ici ?! - la voix de la femme traduisait une colère contenue. (Pélican 254)

On voit que ce sont des contraintes au niveau du discours qui expliquent la présence forte de mais et l'absence fréquente d'un équivalent de mais dans les traductions polonaises. Mais est un marqueur d'espaces discursifs (cf. ci-dessus) et l'attitude d'opposition est rendue en polonais par d'autres expressions. Il y a une tendance à la construire dans le TA comme renforcée ou comme réduite.

6 Nous présentons certaines observations de l'article (sous presse) cité. 


\subsection{LE RENFORCEMENT DE L'OPPOSITION}

2.2.1. par des expressions qui marquent l'évidence :

(13) [Ludo est surpris de voir la voiture de sa mère qui ne vient jamais] - Mais c'est la voiture à ma mère, s'écria Ludo [...] (Noces 212)

(13') - - Ale to przecież samochód mojej matki - wrzasnął Ludo [...] (Barbarzyńskie 139)

L'évidence fonde une opposition suffisamment forte pour que marqueur mais soit même effacé dans la traduction :

(14) Elle [Emma Bovary] se récria, scandalisée.

- Mais, si je vous donne le surplus, répondit effrontément M. Lheureux, n'est-ce pas vous rendre service, à vous ? (MBov 367)

(14') Zaprotestowała oburzona.

- Ø Toż to dla pani wygody, taki mały zapasik - odparł hardo Lheureux. (BovE 263)

Le traducteur choisit de mettre un marqueur d'opposition initial ou bien de commencer par l'expression de l'évidence.

2.2.2. L'explicitation du pronom de la deuxième personne, non exigé par la flexion verbale. La référence se fait en polonais par rapport à la situation d'énonciation, et non par anaphore, et l'emploi du pronom peut servir à renforcer le sens de l'énoncé :

(15) - Mais qu'est-ce que tu me chantes ? (Noces 205)

$\left(15^{\prime}\right) \quad$ - Ale co też ty wygadujesz? (Barbarzyńskie 135)

2.3. L'AFFAIBLISSEMENT OU L'ANNULATION DE L'OPPOSITION PEUT SE FAIRE DE PLUSIEURS MANIÈRES :

- avec l'équivalent lecz, qui implique une opposition intellectuelle sans émotion :

(16) - Emmène-moi ! s'écria-t-elle. Enlève-moi !... Oh ! je t'en supplie ! [...]

- Mais..., reprit Rodolphe. (MBov 276) 
(16') - Zabierz mnie stąd - wykrzyknęła - porwij! Ach, błagam cię! [...] - Lecz... - podjął Rudolf. (BovM 142)

La réplique en polonais laisse comprendre que Rodolphe a procédé à un type de raisonnement, sans se laisser emporter par des émotions ;

- avec les équivalents de la langue parlée qui introduisent une espèce de rectification :

(17) Chez moi, a dit Maixent, on ne me laissera pas aller faire le guignol, tout seul, loin dans la campagne.

- Mais non, a dit Joachim, on fera semblant! On va camper dans le terrain vague! (Petit 35)

(17') - Co ty - powiedział Joachim. - Będziemy tylko udawać! Zrobimy sobie camping na placu! (Mikołajek 29)

A la place d'une expression de la langue parlée, co ty, il est possible de mettre p. ex. też coś, e tam, ale tam; l'opposition est donc faible ;

- avec les expressions de la langue parlée qui mettent en question du point de vue du co-énonciateur, pour qu'il change de sujet : les expressions pertinentes sont p. ex. no, a...; a co z...; no, a co z...; a jak $\operatorname{tam} z$..., no a jak tam $z$... :

(18) Mes notes sont bonnes et je me sens vraiment mordu. [...] - Mais tes amours ? insista-t-elle. (Secret 215)

$\left(18^{\prime}\right) \quad$ - No, a twoje sprawy sercowe? - nie ustępowała. (Sekret 152)

\section{LA CRÉATIVITÉ DU TRADUCTEUR}

Les traducteurs ont proposé des solutions qui s'expliquent par des tendances qui ne sont pas obligatoires. Nous voudrions montrer le 'mot en discours' dans le TA au prisme de la créativité du traducteur, telle qu'elle est conceptualisée par Jerzy Brzozowski (2011).

Il y a des procédés qui relèvent de la routine, adoptés par le traducteur lorsqu'il n'a pas le choix (Brzozowski 2010 : 76). Par conséquent, choisir l'équivalent le plus adéquat relève déjà de la créativité du traducteur. L'auteur parle des 'figures de traduction', qui sont une manifestation de la créativité du traducteur, elles possèdent des marques formelles 
et fonctionnelles (Brzozowski 2011 : 80-81). Les fonctions sont empruntées au modèle de Bühler-Jakobson, et les formes viennent du répertoire classique (Głowiński et al. 2008). Brzozowski discute des cas où, compte tenu des fonctions du langage, on considère comme justifié et attendu le recours à l'explicitation, à la généralisation, à la modulation, à l'adaptation, à l'inversion, à la réduction et à l'amplification. Ces figures sont saisies par rapport à un fond, c'est-à-dire la langue de l'original ou la langue de la traduction. Nous envisageons la langue de l'original.

\subsection{LA FONCTION RÉFÉRENTIELLE}

Nous avons distingué trois cas de figures qui modifient le DCnt de ce point de vue :

- amplification - le traducteur ajoute une information supplémentaire qu'il considère comme importante pour le lecteur. C'est le cas de la traduction du verbe dire par des verbes sémantiquement riches, p. ex. (3'). Il en est de même avec le paraverbal dans $\left(4^{\prime}\right)$, en se levant traduit par podrywając się 'en se levant en sursaut'. Dans $(2$ ') est ajouté un verbe sémantiquement riche dodać 'ajouter'. Pourtant l'amplification peut être aussi une faute de traduction, c'est le cas dans ( $\left.1^{\prime}\right)$ où le même verbe spytać 'demander' figure dans le DCité et le DCnt (1'). Le polonais obéit au principe de la variation dans le choix des mots proches ;

- réduction - dans $\left(8^{\prime}\right)$ le traducteur a supprimé dans le récit la description du paraverbal (le gémissement), dans (10') il a supprimé le pronom qui réfère au co-énonciateur, et dans (11') le DCnt. Dans (10') et $\left(11^{\prime}\right)$, les éléments supprimés sont inférés par le lecteur dans la situation d'énonciation ;

- modulation - un autre point de vue sur le référent est attesté dans $\left(9^{\prime}\right)$. La qualification postposée au DCité n'apporte aucun élément nouveau (le lecteur sait que c'était une question), alors que l'annonce d'une question au niveau du récit dans l'original exprime une certaine stratégie vis-à-vis du lecteur. Un tel changement modifie donc le contrat de lecture.

\subsection{LA FONCTION CONATIVE ET EXPRESSIVE}

Elles dominent dans des répliques introduites par mais. Les figures identifiées sont : 
- amplification par des marqueurs d'évidence dans (13') et (14') qui renforcent l'expressivité, et dans $\left(15^{\prime}\right)$ par la particule też qui a le même rôle. L'amplification dans (15') par le pronom de la $2^{\text {ème }}$ personne intensifie la fonction conative ;

- réduction - dans (16') - l'équivalent lecz est spécialisé dans l'opposition intellectuelle, donc annule les émotions signifiées par mais initial. La fonction expressive est réduite et l'énoncé se présente comme livresque ;

- adaptation - les expressions de la langue parlée dans (17') et (18') introduisent une variation situationnelle absente du TD. L'effacement du marqueur, comme p. ex. dans (14'), peut être vu comme une adaptation à la langue d'accueil.

\section{CONCLUSION}

Les mots dire et mais en discours subissent des contraintes spécifiques lorsqu'ils se trouvent dans le discours littéraire et lorsque celui-ci est traduit en polonais.

Nous avons observé que le verbe dire placé en incise, lorsqu'il n'est pas rendu par des équivalents de base mówić/powiedzieć/rzec, est traduit par les verbes plus riches sémantiquement, qui explicitent le plus souvent la valeur d'acte de l'énoncé. Quand le verbe de dire est implicite, les traducteurs ajoutent souvent dans le TA un verbe de dire. Les verbes de dire en polonais peuvent être coordonnés à des verbes d'action, ce qui montre que les paroles des personnages et le récit ne sont pas distincts comme en français. Le dire peut être qualifié par des éléments puisés dans le récit, qui enrichissent le DCnt. Le co-énonciateur dans l'incise est repéré par la situation d'énonciation et non par un pronom, donc le DCnt est souvent simplifié.

L'opposition introduite par mais est reconstruite de plusieurs manières dans le texte polonais. La présence du marqueur initial est facultative. L'opposition est souvent renforcée par des expressions qui signalent l'évidence ou l'intensification et par l'explicitation du pronom de la $2^{\text {ème }}$ personne du singulier (non obligatoire avec le verbe). L'opposition est réduite, lorsqu'elle sert à rectifier ou à changer de sujet de l'entretien, dans ce cas apparaissent des expressions de la langue parlée, ou familières. 
Ces observations du type contrastif, dans la perspective traductologique montrent l'impact des solutions des traducteurs sur les fonctions du langage, surtout celle référentielle et celle expressive. Les déplacements qui résultent d'un choix parmi de multiples possibilités peuvent être considérés comme la manifestation de la créativité du traducteur. Il y a des cas où le lecteur et l'analyste décèlent des fautes, à cause de la multiplicité des facteurs qui influent sur les choix du traducteur.

\section{RÉFÉRENCES}

Anscombre J.-C., 2009, « Des adverbes d'énonciation aux marqueurs d'attitude énonciative : le cas de la construction tout + adjectif », Langue française 161, 59-80.

Becher V., 2010, «Toward a More Rigorous Treatment of the Explicitation Hypothesis ", in : Translation Studies, "trans-kom" 3, 1, 1-25, http://www.transkom.eu/ihv_03_01_2010.html (consulté le 17 juin 2015)

Bourne J., 2002, «He said, she said. Controling illocutionary force in the translation of literary dialogue », Target 14, 2, 241-261.

Chevalier J-C., Delport M.-F., 1995, Problèmes linguistiques de la traduction, L'horlogerie de Saint Jérôme, Paris.

Danon-Boileau L., 1982, Produire le fictif, Paris.

Dostie G., 2004, Pragmaticalisation et marqueurs discursifs. Analyse sémantique et traitement lexicographique, Bruxelles.

Ducrot O., 1980, Dire et ne pas dire, Paris, deuxième édition corrigée et augmentée.

Durrer S., 1994, Le dialogue romanesque. Style et structure, Genève.

Dutka A., 1992, "A propos des morphèmes proches de ale 'mais' en polonais dans l'approche argumentative », Kwartalnik Neofilologiczny 39, 1, 27-44.

Dutka A., 1993, «Les connecteurs argumentatifs en polonais », in : Dittmar N., Reich A. (éd.), Modality in Language Acquisition. Modalité et acquisition des langues, Berlin, 97-109.

Dutka-Mańkowska A., 2010, « Le discours direct rapporté en français et en polonais dans des textes littéraires », in : Dutka-Mańkowska A., Giermak-Zielińska T. (éd.), Des mots et du texte aux conceptions de la description linguistique, Varsovie, 141-148.

Dutka-Mańkowska A., 2012, «Le discours autre dans la retraduction de Madame Bovary en polonais », in : Kacprzak A., Gajos M. (éd.), Pluralité des cultures : chances ou menaces?, Lask, 117-127.

Dutka-Mańkowska A., (sous presse), «L'attitude énonciative d'opposition introduite par mais - l'approche contrastive français-polonais », Roczniki Humanistyczne.

Głowiński M. et alii, 2008, Słownik terminów literackich, Wrocław. 
Gournay L., 2013, «Traduction des énoncés en incise du discours direct : l'apport de la linguistique contrastive», ELA. Études de linguistique appliquée 172, 397-413.

Guillemin-Flescher J., 1986, «Le linguiste devant la traduction », Fabula 7, 59-68. Guillemin-Flescher J., 2003, «Théoriser la traduction », Revue Française de Linguistique Appliquée 2, 8, 7-18.

Hanote S., 2004, «Des introducteurs de discours aux indices de frayage», in : Lopez-Munoz J.-M., Marnette S., Rosier L. (éd.), Le discours rapporté dans tous ses états, Paris, 538-548.

Kerbrat-Orecchioni C., 2005, Le discours en interaction, Paris.

Mossberg M., Jansson Ghadiri K., 2010, «Les verbes introducteurs dans le incises de citation : étude contrastive français-suédois », Studia Neophilologica 82, 69-90.

Nita R., 2006, "'...' ironise un professeur vs '...', says a teacher ou Sur les dérives du discours direct dans la traduction des verbes introducteurs. Etude contrastive français, anglais, roumain ", in : Delesse C. (éd.), Discours rapporté(s). Approche(s) linguistique(s) et/ou traductologique(s), Arras, 173-195.

Poncharal B., 2003, La représentation des paroles au discours indirect libre en anglais et en français, Paris.

Rabatel A., 2014, «Empathie, points de vue, méta-représentation et dimension cognitive du dialogisme », ELA. Études de linguistique appliquée 173, 27-45.

\section{SOURCES D'EXEMPLES}

Bourdin F., 2010, Le secret de Clara, Paris/2005, Sekret Clary, trad. J. Polachowska, Warszawa.

Chwin S., 2003, Złoty pelikan, Gdańsk/2009, Le Pélican d’or, trad. F. Laurent, Belval.

Duras M., 1958, Moderato cantabile, Paris/1998, Moderato cantabile, trad. Z. Cesul, Warszawa.

Flaubert G., 1857/2014, Madame Bovary, Paris/2005, Pani Bovary. Z obyczajów prowincji, trad. R. Engelking, Gdańsk (BovE)/2008, Pani Bovary, trad. A. Micińska, Kraków (BovM).

Makine A., 2000, Le crime d'Olga Arbelina, Paris/1999, Zbrodnia Olgi Arbeliny, trad. M. Hołdyńska, Warszawa.

Mauriac F., 1925/1978, Le désert de l'amour, in : Mauriac F., Oeuvres romanesques et théatrâles complètes, Paris, t. 1, p. 737-862/1958, Pustynia miłości, trad. S. Zgórska, Warszawa.

Queffélec Y., 1985, Les noces barbares, Paris/1999, Barbarzyńskie zaślubiny, trad. M. Cebo, Warszawa.

Schmitt E.-E., 2007, Odette Toulemonde et autres histoires, Paris/2009, Odette i inne historie miłosne, trad. J. Brzezowski, Kraków. 
Sempé/Goscinny, 2007, Le Petit Nicolas et les copains, Paris/1979, Mikołajek i inne chłopaki, trad. B. Grzegorzewska, Warszawa.

Zola E., 1978, Nana, Paris/1987, Nana, trad. Z. Karczewska-Markiewicz, Warszawa.

\title{
QUELQUES CONTRAINTES DANS LA TRADUCTION DU DISCOURS DIRECT DU FRANÇAIS EN POLONAIS : LE CADRAGE ÉNONCIATIF ET LE CONNECTEUR MAIS AU DÉBUT D'UNE RÉPLIQUE
}

\begin{abstract}
Résumé
L'article présente la traduction des séquences au discours direct, du français vers le polonais, dans quelques romans. L'analyse porte sur le verbe dire dans le discours citant et sur mais au début d'une réplique. Dans le texte d'arrivée, le discours citant tend à enrichir sémantiquement les circonstances de l'énonciation rapportée par le verbe qui en précise les différents aspects et par le déplacement des éléments du récit. La référence à la situation d'énonciation n'explicite pas, comme en français, de paramètres de l'acte d'énonciation, p.ex. l'interlocuteur. Les répliques introduites par mais se caractérisent dans le texte traduit par les expressions liées à l'interaction, et "l'attitude énonciative" (notion de J.-C. Anscombre) d'opposition, exprimée par divers moyens, est intensifiée ou affaiblie par rapport au texte du départ. Les phénomènes sus-mentionnés sont interprétés comme des manifestations de la créativité du traducteur, qui influent surtout sur la fonction référentielle et celle expressive.

Mots-clés : récit, discours direct, attitude énonciative, traduction, connecteur mais

\section{SOME FEATURES OF THE TRANSLATION OF THE DIRECT SPEECH FROM FRENCH TO POLISH: THE ENUNCIATIVE FRAME AND THE CONNECTOR MAIS AT THE BEGINNING OF A REPLY}

\section{Summary}

This article is devoted to the Polish translation of sequences of direct speech in selected French novels. The words under analysis are the verb dire as well as mais. In the case of introductory discourse there is a tendency to enrich the semantic circumstances of the generation of the speech act quoted. There is often a verb specifying the various aspects of this act, the context of the verb is sometimes expanded as well to include elements drawn from the narrative. In the Polish version appears a reference to the situation where in original French the parameters of the act of speaking (for example, the interlocutor), are indicated explicite. Replies starting with mais bring into the translation a wide 
range of expressions related to the interaction and the "enunciative attitude" (the concept of J.-C. Anscombre) of the opposition is expressed by a whole range of measures and in the Polish language is strengthened or weakened. The observed phenomenon is interpreted as a manifestation of the creativity of the translator, especially affecting the referential and expressive function of the target texts.

Key words: story, translation, direct speech, introductory discourse, mais (connector), enunciative attitude 